(C) 2012 IEEE. Personal use of this material is permitted. Permission from IEEE must be obtained for all other uses, in any current or future media, including reprinting/republishing this material for advertising or promotional purposes, creating new collective works, for resale or redistribution to servers or lists, or reuse of any copyrighted component of this work in other works. 


\title{
MMSE-DFE Based MIMO Relay System with Correlated Fading Channel
}

\author{
Yue Rong, Senior Member, IEEE
}

\begin{abstract}
We consider a practical dual-hop nonregenerative multiple-input multiple-output (MIMO) relay system, where the relay node only knows the correlation matrix of the relaydestination channel. A nonlinear minimal mean-squared error (MMSE)-based decision feedback equalizer (DFE) is used at the destination node to retrieve the source signals. We derive the structure of statistically optimal source and relay precoding matrices to minimize a class of objective functions which are multiplicatively Schur-convex with respect to the diagonal elements of the MSE matrix. Simulation results demonstrate that the proposed algorithm has a very close performance compared to MIMO relay system with full channel knowledge at the relay node, and thus is very useful for practical relay systems.
\end{abstract}

Index Terms-Channel correlation, DFE, MIMO relay, MMSE.

\section{INTRODUCTION}

Nonregenerative multiple-input multiple-output (MIMO) relay communication systems have been recently investigated under various assumptions on the availability of channel state information (CSI) [1]-[3]. Assuming that the full CSI of both the source-relay and relay-destination hops is available at the relay node, the optimal relay precoding matrix was developed in [1] and [2]. In [3], the relay precoding matrix was designed to maximize the ergodic source-destination channel capacity where the full source-relay CSI is available, while only the covariance matrix of the relay-destination channel is known at the relay node.

From the aspect of overall system implementation, in a twohop relay system, it is most spectrally efficient to carry out the optimization of the source and relay precoding matrices at the relay node, which requires the least amount of CSI/precoding matrices feedback among different nodes. Under this scenario, in this letter, we make the practical assumption that the full source-relay CSI is available at the relay node through channel training, while the full CSI of the relay-destination hop is only available at the destination node. Moreover, the covariance matrix of the relay-destination channel is available at the relay node through a low-rate feedback from the destination node.

Due to its excellent bit-error-rate (BER) performance, a nonlinear minimal mean-squared error (MMSE) based decision feedback equalizer (DFE) [4] is used at the destination node to retrieve the source signals. We derive the structure of statistically optimal source and relay precoding matrices to minimize a class of objective functions which are multiplicatively Schur-convex [5] with respect to the diagonal elements of the MSE matrix. We would like to mention that for

Y. Rong is with the Department of Electrical and Computer Engineering, Curtin University, Bentley, WA 6102, Australia (e-mail: y.rong@curtin.edu.au). This work was supported under the Australian Research Council's Discovery Projects funding scheme (project numbers DP110100736, DP110102076). point-to-point (single-hop) MIMO system, statistically optimal source precoding matrix design was recently addressed in [6] using the zero-forcing (ZF)-DFE receiver. However, our work is the first one in this direction for MIMO relay systems using the MMSE-DFE receiver. Simulation results demonstrate that the proposed algorithm yields much smaller BER than MIMO relay systems using linear receivers, and has a very close performance compared to MIMO relay system with full channel knowledge at the relay node [7]. Thus, the proposed algorithm is very useful for practical MIMO relay communication systems.

\section{System MODEL}

We consider a three-node two-hop MIMO communication system where the source node (node 1) transmits information to the destination node (node 3 ) with the aid of one relay node (node 2). The $i$ th node is equipped with $N_{i}, i=1,2,3$, antennas. We focus on the case where the direct link between the source and destination nodes is sufficiently weak to be ignored as in [1]-[3]. This scenario occurs when the direct link is blocked by an obstacle such as a mountain. Using the nonregenerative relay strategy as in [1]-[3], the received signal vector at the destination node can be written as

$$
\mathbf{y}=\mathbf{H}_{2} \mathbf{F}_{2} \mathbf{H}_{1} \mathbf{F}_{1} \mathbf{s}+\mathbf{H}_{2} \mathbf{F}_{2} \mathbf{v}_{2}+\mathbf{v}_{3} \triangleq \overline{\mathbf{H}} \mathbf{s}+\overline{\mathbf{v}}
$$

where $\mathbf{s}$ is the $N_{b} \times 1$ source signal vector, $\mathbf{F}_{1}$ is the $N_{1} \times N_{b}$ source precoding matrix, $\mathbf{H}_{1}$ is the $N_{2} \times N_{1}$ MIMO fading channel matrix between the source and relay nodes, $\mathbf{F}_{2}$ is the $N_{2} \times N_{2}$ relay precoding matrix, $\mathbf{H}_{2}$ is the $N_{3} \times N_{2}$ MIMO fading channel matrix between the relay and destination nodes, $\mathbf{v}_{2}$ is an $N_{2} \times 1$ noise vector at the relay node, and $\mathbf{v}_{3}$ is an $N_{3} \times 1$ noise vector at the destination node. Here $\overline{\mathbf{H}} \triangleq \mathbf{H}_{2} \mathbf{F}_{2} \mathbf{H}_{1} \mathbf{F}_{1}$ is the equivalent source-destination MIMO channel, and $\overline{\mathbf{v}} \triangleq \mathbf{H}_{2} \mathbf{F}_{2} \mathbf{v}_{2}+\mathbf{v}_{3}$ is the effective noise vector at the destination node. We assume that $\mathrm{E}\left[\mathbf{s s}^{H}\right]=\mathbf{I}_{N_{b}}$, where $\mathrm{E}[\cdot]$ denotes statistical expectation, $\mathbf{I}_{n}$ denotes an $n \times n$ identity matrix, and $(\cdot)^{H}$ stands for matrix (vector) Hermitian transpose. All noises are independent and identically distributed (i.i.d.) additive white Gaussian noise (AWGN) with zero mean and unit variance.

We assume that the relay node and the destination node know the full CSI of $\mathbf{H}_{1}$ and $\overline{\mathbf{H}}$ respectively through channel training. We also assume that $\mathbf{H}_{2}$ is correlated at the relay side such that $\mathbf{H}_{2}=\mathbf{H}_{w} \mathbf{C}_{2}^{\frac{T}{2}}$ [3], [8], where $\mathbf{H}_{w}$ is a Gaussian random matrix having i.i.d. entries with zero mean and unit variance, and $(\cdot)^{T}$ stands for matrix (vector) transpose. Here $\mathbf{H}_{w}$ is unknown at the relay node, $\mathbf{C}_{2}=\mathbf{C}_{2}^{\frac{1}{2}} \mathbf{C}_{2}^{\frac{H}{2}}$ is the channel correlation matrix which is known at the relay node through a low-rate feedback from the destination node. This scenario occurs, for example, in downlink MIMO relay communication, 
where the source node (base station) and the relay node are static, while the destination node (user terminal) is mobile. In such system, $\mathbf{H}_{1}$ is quasi-static and can be estimated at the relay node, while $\mathbf{H}_{2}$ is time-varying and only its statistics is known at the relay node.

In the example above, since destination nodes usually have limited computation capability, the task of joint source and relay precoding matrices design can not be performed at destination nodes. Moreover, even if the source and relay matrices can be designed at the destination node, then the destination node needs to feed back the source and relay matrices to the source node and relay node, respectively. This is less spectrally efficient compared with the approach where the source and relay matrices are designed at the relay node, and only the source matrix needs to be fed back. Therefore, in this letter, we assume that the optimization of precoding matrices $\mathbf{F}_{1}$ and $\mathbf{F}_{2}$ is carried out at the relay node, and the optimal $\mathbf{F}_{1}$ is fed back from the relay node to the source node.

Using a nonlinear MMSE-DFE receiver at the destination node and assuming that there is no error propagation effect, the $k$ th source symbol is estimated as [4, Ch.8]

$$
\hat{s}_{N_{b}}=\mathbf{w}_{N_{b}}^{H} \mathbf{y}, \hat{s}_{k}=\mathbf{w}_{k}^{H} \mathbf{y}-\sum_{l=k+1}^{N_{b}} b_{k, l} s_{l}, k=1, \cdots, N_{b}-1
$$

where $\mathbf{w}_{k}$ is the feed-forward vector for the $k$ th symbol, and $b_{k, l}, l=k+1, \cdots, N_{b}$, are the feedback coefficients for the $k$ th symbol. By introducing $\mathbf{W}=\left[\mathbf{w}_{1}, \mathbf{w}_{2}, \cdots, \mathbf{w}_{N_{b}}\right]$, $\hat{\mathbf{s}}=\left[\hat{s}_{1}, \hat{s}_{2}, \cdots, \hat{s}_{N_{b}}\right]^{T}$ and an $N_{b} \times N_{b}$ strictly upper-triangle matrix B with nonzero elements $b_{k, l}$, we can represent (2) in matrix form as

$$
\hat{\mathbf{s}}=\mathbf{W}^{H} \mathbf{y}-\mathbf{B} \mathbf{s}=\left(\mathbf{W}^{H} \overline{\mathbf{H}}-\mathbf{B}\right) \mathbf{s}+\mathbf{W}^{H} \overline{\mathbf{v}} .
$$

Here $\mathbf{W}$ and $\mathbf{B}$ are the feed-forward and feedback matrices of the DFE receiver, respectively. From (3), the MSE matrix of the signal waveform estimation is given by

$$
\begin{aligned}
\mathbf{E} & =\mathrm{E}\left[(\hat{\mathbf{s}}-\mathbf{s})(\hat{\mathbf{s}}-\mathbf{s})^{H}\right] \\
& =\left(\mathbf{W}^{H} \overline{\mathbf{H}}-\mathbf{B}-\mathbf{I}_{N_{b}}\right)\left(\mathbf{W}^{H} \overline{\mathbf{H}}-\mathbf{B}-\mathbf{I}_{N_{b}}\right)^{H}+\mathbf{W}^{H} \mathbf{C}_{\bar{v}} \mathbf{W}
\end{aligned}
$$

where $\mathbf{C}_{\bar{v}} \triangleq \mathbf{H}_{2} \mathbf{F}_{2} \mathbf{F}_{2}^{H} \mathbf{H}_{2}^{H}+\mathbf{I}_{N_{3}}$ is the covariance matrix of $\overline{\mathbf{v}}$. [9]

Let us introduce the following matrix $\mathrm{QR}$ decomposition

$$
\mathbf{G} \triangleq\left[\begin{array}{c}
\mathbf{C}_{\overline{\mathbf{v}}}^{-\frac{1}{2}} \overline{\mathbf{H}} \\
\mathbf{I}_{N_{b}}
\end{array}\right]=\mathbf{Q R}=\left[\begin{array}{l}
\overline{\mathbf{Q}} \\
\mathbf{Q}
\end{array}\right] \mathbf{R}
$$

where $\mathbf{R}$ is an $N_{b} \times N_{b}$ upper-triangular matrix with all positive diagonal elements, $\mathbf{Q}$ is an $\left(N_{b}+N_{3}\right) \times N_{b}$ semi-unitary matrix with $\mathbf{Q}^{H} \mathbf{Q}=\mathbf{I}_{N_{b}}, \overline{\mathbf{Q}}$ is a matrix containing the first $N_{3}$ rows of $\mathbf{Q}$, and $\mathbf{Q}$ contains the last $N_{b}$ rows of $\mathbf{Q}$. It is shown in [7] that the optimal $\mathbf{W}, \mathbf{B}$, and $\mathbf{E}$ are given by

$$
\mathbf{W}=\mathbf{C}_{\bar{v}}^{-\frac{1}{2}} \overline{\mathbf{Q}} \mathbf{D}_{R}^{-1}, \quad \mathbf{B}=\mathbf{D}_{R}^{-1} \mathbf{R}-\mathbf{I}_{N_{b}}, \quad \mathbf{E}=\mathbf{D}_{R}^{-2}
$$

where $(\cdot)^{-1}$ denotes matrix inversion, and for a matrix $\mathbf{A}$, $\mathbf{D}_{A}$ is a matrix taking the diagonal elements of $\mathbf{A}$ as the main diagonal and zero elsewhere.

We would like to mention that when error propagation is considered, it is intractable to derive a closed form expression for the optimal $\mathbf{W}, \mathbf{B}$, and $\mathbf{E}$. The error propagation effect increases the system BER and will be studied in Section IV.

\section{Proposed Algorithm}

We consider a class of objective functions $f$ which are multiplicatively Schur-convex [5] with respect to the main diagonal elements of $\mathbf{E}$ (or equivalently $\mathbf{D}_{R}^{-2}$ base on (4)). A list of multiplicatively Schur-convex functions that are commonly used in MIMO communication system design can be found in [10, Ch.4] such as the sum of MSEs $f\left(\mathbf{d}\left[\mathbf{D}_{R}^{-2}\right]\right)=\sum_{i=1}^{N_{b}}\left[\mathbf{D}_{R}^{-2}\right]_{i, i}$ and the product of MSEs $f\left(\mathbf{d}\left[\mathbf{D}_{R}^{-2}\right]\right)=\prod_{i=1}^{N_{b}}\left[\mathbf{D}_{R}^{-2}\right]_{i, i}$. Here for a matrix $\mathbf{A},[\mathbf{A}]_{i, i}$ denotes the $i$ th main diagonal element of $\mathbf{A}$, and $\mathbf{d}[\mathbf{A}]$ is a column vector containing all main diagonal elements of $\mathbf{A}$. It has been shown in [7] that when the full CSI of $\mathbf{H}_{2}$ is available at the relay node, the source and relay precoding matrices optimization problem can be written as

$$
\begin{array}{rl}
\min _{\mathbf{F}_{1}, \mathbf{F}_{2}} & f\left(\mathbf{d}\left[\mathbf{D}_{R}^{-2}\right]\right) \\
\text { s.t. } & \mathbf{I}_{N_{b}}+\overline{\mathbf{H}}^{H} \mathbf{C}_{\bar{v}}^{-1} \overline{\mathbf{H}}=\mathbf{R}^{H} \mathbf{R} \\
& \operatorname{tr}\left(\mathbf{F}_{2}\left(\mathbf{H}_{1} \mathbf{F}_{1} \mathbf{F}_{1}^{H} \mathbf{H}_{1}^{H}+\mathbf{I}_{N_{2}}\right) \mathbf{F}_{2}^{H}\right) \leq P_{2} \\
& \operatorname{tr}\left(\mathbf{F}_{1} \mathbf{F}_{1}^{H}\right) \leq P_{1}
\end{array}
$$

where $\operatorname{tr}(\cdot)$ denotes matrix trace, $P_{i}>0, i=1,2$, is the transmission power available at the $i$ th node, (7) and (8) represent the transmission power constraint at the relay node and the source node, respectively.

However, when the full $\mathbf{H}_{2}$ knowledge is not available at the relay node, the Cholesky decomposition constraint in (6) can not be guaranteed since matrix $\mathbf{M} \triangleq \mathbf{I}_{N_{b}}+\overline{\mathbf{H}}^{H} \mathbf{C}_{\bar{v}}^{-1} \overline{\mathbf{H}}$ is unknown. To make the problem trackable, we consider $\mathrm{E}_{\mathbf{H}_{2}}[\mathbf{M}]$, where the expectation is performed over the realization of $\mathbf{H}_{2}$. Now the source and relay precoding matrices optimization problem is given by

$$
\begin{array}{rl}
\min _{\mathbf{F}_{1}, \mathbf{F}_{2}} & f\left(\mathbf{d}\left[\mathbf{D}_{\breve{R}}^{-2}\right]\right) \\
\text { s.t. } & \mathrm{E}_{\mathbf{H}_{2}}[\mathbf{M}]=\breve{\mathbf{R}}^{H} \breve{\mathbf{R}} \\
& \operatorname{tr}\left(\mathbf{F}_{2}\left(\mathbf{H}_{1} \mathbf{F}_{1} \mathbf{F}_{1}^{H} \mathbf{H}_{1}^{H}+\mathbf{I}_{N_{2}}\right) \mathbf{F}_{2}^{H}\right) \leq P_{2} \\
& \operatorname{tr}\left(\mathbf{F}_{1} \mathbf{F}_{1}^{H}\right) \leq P_{1} .
\end{array}
$$

By using the matrix inversion lemma, we obtain $\left(\mathbf{I}_{N_{2}}+\mathbf{F}_{2}^{H} \mathbf{H}_{2}^{H} \mathbf{H}_{2} \mathbf{F}_{2}\right)^{-1}=\mathbf{I}_{N_{2}}-\mathbf{F}_{2}^{H} \mathbf{H}_{2}^{H}\left(\mathbf{H}_{2} \mathbf{F}_{2} \mathbf{F}_{2}^{H} \mathbf{H}_{2}^{H}+\right.$ $\left.\mathbf{I}_{N_{3}}\right)^{-1} \mathbf{H}_{2} \mathbf{F}_{2}$. Therefore, there is $\mathbf{F}_{2}^{H} \mathbf{H}_{2}^{H} \mathbf{C}_{\bar{v}}^{-1} \mathbf{H}_{2} \mathbf{F}_{2}=\mathbf{I}_{N_{2}}-$ $\left(\mathbf{I}_{N_{2}}+\mathbf{F}_{2}^{H} \mathbf{H}_{2}^{H} \mathbf{H}_{2} \mathbf{F}_{2}\right)^{-1}$, and we have

$$
\begin{aligned}
\mathrm{E}_{\mathbf{H}_{2}}[\mathbf{M}] & =\mathrm{E}_{\mathbf{H}_{2}}\left[\mathbf{I}_{N_{b}}+\mathbf{F}_{1}^{H} \mathbf{H}_{1}^{H}\right. \\
& \left.\times\left(\mathbf{I}_{N_{2}}-\left(\mathbf{I}_{N_{2}}+\mathbf{F}_{2}^{H} \mathbf{H}_{2}^{H} \mathbf{H}_{2} \mathbf{F}_{2}\right)^{-1}\right) \mathbf{H}_{1} \mathbf{F}_{1}\right] .
\end{aligned}
$$

Due to the matrix inversion operation, the exact closed form expression of (13) is difficult to obtain. To avoid this difficulty, we resort to a close approximation of (13). Since $\mathbf{X}^{-1}$ is a matrix convex function of $\mathbf{X}$ [5], an upper-bound of (13) can be obtained as

$$
\begin{aligned}
& \mathrm{E}_{\mathbf{H}_{2}}[\mathbf{M}] \\
\leq & \mathbf{I}_{N_{b}}+\mathbf{F}_{1}^{H} \mathbf{H}_{1}^{H}\left(\mathbf{I}_{N_{2}}-\left(\mathbf{I}_{N_{2}}+\mathbf{F}_{2}^{H} \mathrm{E}_{\mathbf{H}_{2}}\left[\mathbf{H}_{2}^{H} \mathbf{H}_{2}\right] \mathbf{F}_{2}\right)^{-1}\right) \mathbf{H}_{1} \mathbf{F}_{1} \\
= & \mathbf{I}_{N_{b}}+\mathbf{F}_{1}^{H} \mathbf{H}_{1}^{H}\left(\mathbf{I}_{N_{2}}-\left(\mathbf{I}_{N_{2}}+\mathbf{F}_{2}^{H} \tilde{\mathbf{C}}_{2} \mathbf{F}_{2}\right)^{-1}\right) \mathbf{H}_{1} \mathbf{F}_{1} \\
\triangleq & \tilde{\mathbf{M}}
\end{aligned}
$$


where $\tilde{\mathbf{C}}_{2} \triangleq N_{3} \mathbf{C}_{2}^{T}$, and for two matrices $\mathbf{A}$ and $\mathbf{B}, \mathbf{A} \leq \mathbf{B}$ means that $\mathbf{B}-\mathbf{A}$ is a positive semi-definite matrix.

The comparison of the upper-bound (14) and the true value (13) is shown in Fig. 1 in terms of their traces $\operatorname{tr}(\tilde{\mathbf{M}})$, $\mathrm{E}_{\mathbf{H}_{2}}[\operatorname{tr}(\mathbf{M})]$ and their $(2,2)$-th elements $[\tilde{\mathbf{M}}]_{2,2}, \mathrm{E}_{\mathbf{H}_{2}}\left[[\mathbf{M}]_{2,2}\right]$ versus $P_{1}$. Here we set $N_{b}=3, N_{i}=N=3, i=1,2,3$, $\mathbf{F}_{1}=\sqrt{P_{1} / N_{1}} \mathbf{I}_{N_{1}}$, and $\mathbf{F}_{2}=\sqrt{P_{2} / N_{2}} \mathbf{I}_{N_{2}}$ with $P_{2}=20 \mathrm{~dB}$. The value of $\mathrm{E}_{\mathbf{H}_{2}}[\operatorname{tr}(\mathbf{M})]$ and $\mathrm{E}_{\mathbf{H}_{2}}\left[[\mathbf{M}]_{2,2}\right]$ is obtained from using $10^{4}$ random realizations of $\mathbf{H}_{2}$. Based on [8], we assume that $\mathbf{C}_{2}$ has the commonly used exponential Toeplitz structure such that

$$
\left[\mathbf{C}_{2}\right]_{m, n}=\mathcal{J}_{0}\left(2 \pi|m-n| / c_{2}\right)
$$

where $\mathcal{J}_{0}(\cdot)$ is the zeroth order Bessel function of the first kind, and $c_{2}$ stands for the correlation coefficient which depends on physical factors such as the angle of arrival spread, spacing between antenna elements, and the wavelength at the center frequency [8]. Here we set $c_{2}=10$. It can be seen from Fig. 1 that $\operatorname{tr}(\tilde{\mathbf{M}})$ and $[\tilde{\mathbf{M}}]_{2,2}$ are very close to $\mathrm{E}_{\mathbf{H}_{2}}[\operatorname{tr}(\mathbf{M})]$ and $\mathrm{E}_{\mathbf{H}_{2}}\left[[\mathbf{M}]_{2,2}\right]$, respectively, indicating that the upper-bound (14) is indeed very tight. We would like to mention that the tightness of (14) is observed for different $\mathbf{F}_{1}$, $\mathbf{F}_{2}$, and $\mathbf{C}_{2}$.

In the following, by using (14), we rewrite the source and relay precoding matrices design problem as

$$
\begin{array}{rl}
\min _{\mathbf{F}_{1}, \mathbf{F}_{2}} & f\left(\mathbf{d}\left[\mathbf{D}_{\tilde{R}}^{-2}\right]\right) \\
\text { s.t. } & \tilde{\mathbf{M}}=\tilde{\mathbf{R}}^{H} \tilde{\mathbf{R}} \\
& \operatorname{tr}\left(\mathbf{F}_{2}\left(\mathbf{H}_{1} \mathbf{F}_{1} \mathbf{F}_{1}^{H} \mathbf{H}_{1}^{H}+\mathbf{I}_{N_{2}}\right) \mathbf{F}_{2}^{H}\right) \leq P_{2} \\
& \operatorname{tr}\left(\mathbf{F}_{1} \mathbf{F}_{1}^{H}\right) \leq P_{1}
\end{array}
$$

where $\tilde{\mathbf{R}}$ is an $N_{b} \times N_{b}$ upper-triangular matrix. Let us introduce $\mathbf{H}_{1}=\mathbf{U}_{1} \boldsymbol{\Sigma}_{1} \mathbf{V}_{1}^{H}$ as the singularvalue decomposition (SVD) of $\mathbf{H}_{1}, \tilde{\mathbf{C}}_{2}=\mathbf{U}_{2} \boldsymbol{\Sigma}_{2}^{2} \mathbf{U}_{2}^{H}$ as the eigenvalue decomposition (EVD) of $\tilde{\mathbf{C}}_{2}$, and $M \triangleq \min \left(\operatorname{rank}\left(\mathbf{H}_{1}\right), \operatorname{rank}\left(\mathbf{H}_{2}\right), N_{b}\right)$, where $\operatorname{rank}(\cdot)$ denotes the rank of a matrix. We assume that the main diagonal elements in $\boldsymbol{\Sigma}_{1}$ and $\boldsymbol{\Sigma}_{2}$ are arranged in an increasing order, respectively. For any multiplicatively Schurconvex $f$ with respect to $\mathbf{d}\left[\mathbf{D}_{\tilde{R}}^{-2}\right]$, the following theorem is in order.

THEOREM 1: The optimal structure of $\mathbf{F}_{1}$ and $\mathbf{F}_{2}$ as the solution to the problem (16)-(19) in the form of their SVDs is given by

$$
\mathbf{F}_{1}=\tilde{\mathbf{V}}_{1} \boldsymbol{\Lambda}_{1} \mathbf{V}_{F_{1}}^{H}, \quad \mathbf{F}_{2}=\tilde{\mathbf{U}}_{2} \boldsymbol{\Lambda}_{2} \tilde{\mathbf{U}}_{1}^{H}
$$

where $\tilde{\mathbf{V}}_{1}, \tilde{\mathbf{U}}_{1}, \tilde{\mathbf{U}}_{2}$ contain $M$ columns of $\mathbf{V}_{1}, \mathbf{U}_{1}, \mathbf{U}_{2}$ associated with the largest $M$ singular (eigen)-values, $\boldsymbol{\Lambda}_{1}$ and $\boldsymbol{\Lambda}_{2}$ are $M \times M$ diagonal matrices, and $\mathbf{V}_{F_{1}}$ is an $N_{b} \times M$ (semi)-unitary matrix with $\mathbf{V}_{F_{1}}^{H} \mathbf{V}_{F_{1}}=\mathbf{I}_{M}$ and is chosen such that $\mathbf{d}\left[\mathbf{D}_{\tilde{R}}\right]$ has equal entries.

Proof: Introducing $\tilde{\mathbf{C}}_{2}=\tilde{\mathbf{C}}_{2}^{\frac{H}{2}} \tilde{\mathbf{C}}_{2}^{\frac{1}{2}}$ (with $\tilde{\mathbf{C}}^{\frac{1}{2}}=\mathbf{U}_{2} \boldsymbol{\Sigma}_{2} \mathbf{U}_{2}^{H}$ ) and applying the matrix inversion lemma to (14), we have

$$
\tilde{\mathbf{M}}=\mathbf{I}_{N_{b}}+\tilde{\mathbf{H}}^{H} \mathbf{C}_{\tilde{v}}^{-1} \tilde{\mathbf{H}}=\tilde{\mathbf{G}}^{H} \tilde{\mathbf{G}}
$$

where $\tilde{\mathbf{H}} \triangleq \tilde{\mathbf{C}}_{2}^{\frac{1}{2}} \mathbf{F}_{2} \mathbf{H}_{1} \mathbf{F}_{1}, \mathbf{C}_{\tilde{v}} \triangleq \tilde{\mathbf{C}}_{2}^{\frac{1}{2}} \mathbf{F}_{2} \mathbf{F}_{2}^{H} \tilde{\mathbf{C}}_{2}^{\frac{H}{2}}+\mathbf{I}_{N_{2}}$, and $\tilde{\mathbf{G}} \triangleq\left[\tilde{\mathbf{H}}^{H} \mathbf{C}_{\tilde{v}}^{-\frac{H}{2}}, \mathbf{I}_{N_{b}}\right]^{H}$. Using (21), constraint (17) can be equivalently represented as the $\mathrm{QR}$ decomposition constraint on $\tilde{\mathbf{G}}$ as

$$
\tilde{\mathbf{G}}=\tilde{\mathbf{Q}} \tilde{\mathbf{R}} .
$$

Now the problem (16)-(19) is converted to a problem composed of (16), (22), (18), (19). The optimal structure of $\mathbf{F}_{1}$ and $\mathbf{F}_{2}$ for the latter problem has been proven in [7] and is given by (20).

Interestingly, the optimal structure of the source and relay precoding matrices (20) is similar to that of the relay system with the full CSI of $\mathbf{H}_{2}$ at the relay node [7]. The major difference is that $\mathbf{F}_{2}$ is matched to $\mathbf{U}_{2}$, rather than the right singular vector matrix of $\mathbf{H}_{2}$. Since the upper-bound (14) is tight, the structure of $\mathbf{F}_{1}$ and $\mathbf{F}_{2}$ in (20) provides a good solution to the problem (9)-(12).

Using (20) and following the steps in [7], for any multiplicatively Schur-convex $f$, the problem (16)-(19) can be equivalently converted to the following problem

$$
\begin{aligned}
\min _{\boldsymbol{\lambda}_{1}, \boldsymbol{\lambda}_{2}} & -\sum_{k=1}^{M} \log _{2}\left(1+\frac{\lambda_{1, k}^{2} \sigma_{1, k}^{2} \lambda_{2, k}^{2} \sigma_{2, k}^{2}}{1+\lambda_{2, k}^{2} \sigma_{2, k}^{2}}\right) \\
\text { s.t. } & \sum_{k=1}^{M} \lambda_{2, k}^{2}\left(\lambda_{1, k}^{2} \sigma_{1, k}^{2}+1\right) \leq P_{2} \\
& \sum_{k=1}^{M} \lambda_{1, k}^{2} \leq P_{1}
\end{aligned}
$$

where $\boldsymbol{\lambda}_{i} \triangleq\left[\lambda_{i, 1}, \cdots, \lambda_{i, M}\right]^{T}, i=1,2, \lambda_{i, k}$ and $\sigma_{i, k}$ are the $k$ th main diagonal element of $\boldsymbol{\Lambda}_{i}$ and $\boldsymbol{\Sigma}_{i}, i=1,2$, respectively. Note that the optimization problem with matrix variables (16)(19) has been simplified to a problem with scalar variables. The problem (23)-(25) can be efficiently solved by the iterative water-filling algorithm developed in [2]. Finally, $\mathbf{V}_{F_{1}}$ should be chosen such that $\mathbf{d}\left[\mathbf{D}_{\tilde{R}}\right]$ has equal entries. Such $\mathbf{V}_{F_{1}}$ can be efficiently computed using the numerical method developed in [11].

\section{NumericAl EXAMPleS}

In this section, we study the performance of the proposed source and relay precoding matrices through numerical simulations. For simplicity, we consider $N_{i}=N, i=1,2,3$, but the extension to the general case where different nodes have different number of antennas is straightforward. Channel matrices $\mathbf{H}_{1}$ and $\mathbf{H}_{w}$ have i.i.d. Gaussian entries with zeromean and variance $1 / N$. In each channel realization, 2000 randomly generated bits are transmitted by the source node using the QPSK modulation scheme. All simulation results are averaged over 1000 independent channel realizations.

We compare the proposed algorithm with the algorithm developed in [7] where an MMSE-DFE receiver is used at the destination node, the maximal mutual information (MMI) algorithm, and the MMSE algorithm developed in [2] where a linear receiver is used at the destination node. Note that all algorithms in [2] and [7] require full $\mathbf{H}_{2}$ knowledge at the relay node. While for the proposed algorithm, only the knowledge of $\mathbf{C}_{2}$ is necessary at the relay node. For the proposed algorithm and the algorithm in [7], we study their BER 
performance when an ideal (perfect) MMSE-DFE receiver and a practical MMSE-DFE receiver (with error propagation) is used, respectively.

In our first example, we set $N=N_{b}=4$. The channel correlation coefficient ${ }^{1}$ is chosen as $c_{2}=10$ in (15). Fig. 2 shows the BER comparison among four algorithms versus $P_{2}$ for a fixed $P_{1}=20 \mathrm{~dB}$. In the second example, we simulate a MIMO relay system with $N=5, N_{b}=4$, and $c_{2}=10$. The BER performance of four algorithms is displayed in Fig. 3 versus $P_{1}$ for a fixed $P_{2}=20 \mathrm{~dB}$. It can be clearly seen from Figs. 2 and 3 that due to the application of MMSE-DFE receiver, the performance of the proposed algorithm is much better than the algorithms in [2]. In fact, the performance of the proposed algorithm is very close to that of the algorithm in [7] in low to medium $P_{2}$ or $P_{1}$. At high power level, the performance of the proposed algorithm is comparable to that in [7]. Therefore, the proposed algorithm is very useful for practical MIMO relay communication systems.

It can also be seen from Figs. 2 and 3 that for the proposed algorithm and the algorithm in [7], there is an SNR gap between the BER performance of the system using an ideal MMSE-DFE receiver and that using a practical MMSE-DFE receiver. However, even with the error-propagation effect, the BER of the proposed algorithm is still much lower than that of the MMI and MMSE algorithms in [2]. The reason is that although the error propagation reduces the SNR gain, it does not affect the diversity gain of the MMSE-DFE receiver, which is more important in MIMO systems, especially at high SNR region.

We observe in Fig. 2 that the difference between the proposed algorithm and that of [7] is almost constant when $P_{2}$ is above $17 \mathrm{~dB}$. While seen from Fig. 3, the difference between two algorithms increases with $P_{1}$. This indicates that at a fixed $P_{2}$ level, the impact of the difference between $\mathbf{F}_{2}$ using the proposed algorithm and the $\mathbf{F}_{2}$ from [7] on the BER performance increases with $P_{1}$.

\section{Conclusions}

We have derived the optimal structure of the source and relay precoding matrices of a two-hop MIMO relay system with only statistical CSI of $\mathbf{H}_{2}$ at the relay node. A class of multiplicatively Schur-convex objective functions are optimized when an MMSE-DFE receiver is used at the destination node. Simulation results show that the proposed algorithm has a close performance to that of MIMO relay system with full $\mathbf{H}_{2}$ knowledge at the relay node.

\section{REFERENCES}

[1] X. Tang and Y. Hua, "Optimal design of non-regenerative MIMO wireless relays," IEEE Trans. Wireless Commun., vol. 6, pp. 1398-1407, Apr. 2007.

[2] Y. Rong, X. Tang, and Y. Hua, "A unified framework for optimizing linear non-regenerative multicarrier MIMO relay communication systems," IEEE Trans. Signal Process., vol. 57, pp. 4837-4851, Dec. 2009.

${ }^{1}$ We observed that for both the proposed algorithm and the algorithm developed in [7], the system BER increases with an increasing $c_{2}$, due to loss of effective spatial diversity.
[3] C. Jeong and H.-M. Kim, "Precoder design of non-regenerative relays with covariance feedback," IEEE Commun. Lett., vol. 13, pp. 920-922, Dec. 2009.

[4] D. Tse and P. Viswanath, Fundamentals of Wireless Communication. Cambridge University Press, 2005.

[5] A. W. Marshall, I. Olkin, and B. C. Arnold, Inequalities: Theory of Majorization and Its Applications. 2nd Ed., Springer, 2009.

[6] S. Järmyr, B. Ottersten, and E. A. Jorswieck, "Statistical precoding with decision feedback equalization over a correlated MIMO channel," IEEE Trans. Signal Process., vol. 58, pp. 6298-6311, Dec. 2010.

[7] Y. Rong, "Optimal linear non-regenerative multi-hop MIMO relays with MMSE-DFE receiver at the destination," IEEE Trans. Wireless Commun., vol. 9, pp. 2268-2279, Jul. 2010.

[8] D. S. Shiu, G. Foschini, M. Gans, and J. Kahn, "Fading correlation and its effect on the capacity of multielement antenna systems," IEEE Trans. Commun., vol. 48, pp. 503-513, Mar. 2000.

[9] R. A. Horn and C. R. Johnson, Matrix Analysis. Cambridge University Press, 1985.

[10] D. P. Palomar and Y. Jiang, MIMO Transceiver Design via Majorization Theory. now Publishers, 2007.

[11] Y. Jiang, W. Hager, and J. Li, "The generalized triangular decomposition," Mathematics of Computation, vol. 77, pp. 1037-1056, Apr. 2008

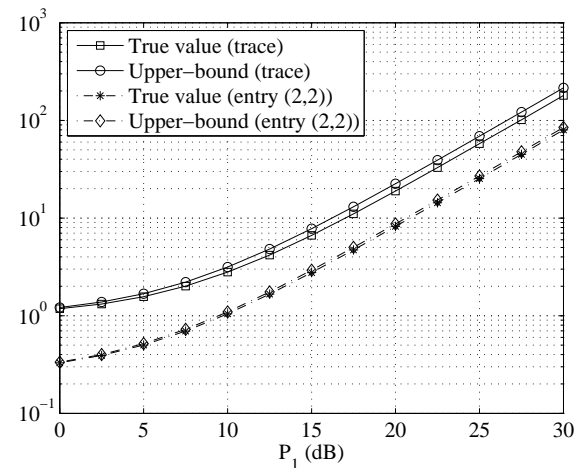

Fig. 1. Comparison between the upper-bound $\operatorname{tr}(\tilde{\mathbf{M}}),[\tilde{\mathbf{M}}]_{2,2}$ and the true value $\mathrm{E}_{\mathbf{H}_{2}}[\operatorname{tr}(\mathbf{M})], \mathrm{E}_{\mathbf{H}_{2}}\left[[\mathbf{M}]_{2,2}\right] \cdot N=N_{b}=3, c_{2}=10$, and $P_{2}=$ $20 \mathrm{~dB}$.

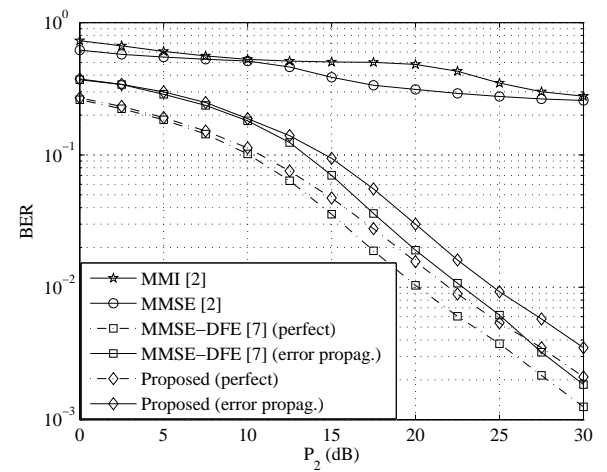

Fig. 2. Example 1: BER versus $P_{2} \cdot N=N_{b}=4, c_{2}=10$, and $P_{1}=20 \mathrm{~dB}$.

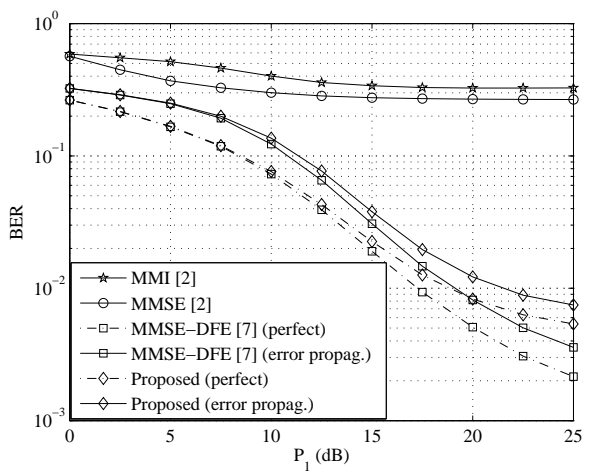

Fig. 3. Example 2: BER versus $P_{1} . N=5, N_{b}=4, c_{2}=10$, and $P_{2}=20 \mathrm{~dB}$ 